\title{
Lymphocytic Colitis Associated to Systemic Lupus Erythematosus in a Young Woman
}

\author{
Salem Bouomrani ${ }^{1,2, *}$, Souad Yahyaoui ${ }^{1}$, Mouna Guermazi ${ }^{1,2}$, Nesrine Regaïeg ${ }^{1,2}$ \\ ${ }^{1}$ Department of Internal medicine, Military Hospital of Gabes, Gabes 6000, Tunisia \\ ${ }^{2}$ Sfax Faculty of Medicine, University of Sfax, Sfax 3029, Tunisia \\ *Corresponding author: salembouomrani@yahoo.fr
}

Received June 24, 2019; Revised August 12, 2019; Accepted August 21, 2019

\begin{abstract}
Introduction: Gastrointestinal symptoms are common in systemic lupus erythematosus (SLE) and are often difficult to interpret. Specific lupus involvement (lupus enteritis) typically affects the jejunum and ileum, whereas colonic and rectal involvement remains rare. Lymphocytic colitis (LC) associated with SLE remains exceptional and unusual. We report an original observation of LC occurring in a 35-year-old patient with SLE. Case report: 35-year-old woman, having in her medical history a Hashimoto thyroiditis diagnosed for four years and a SLE diagnosed for three years, consulted for chronic fluid diarrhea, not improved by symptomatic treatment, and associated for two weeks with rectorrhagia. Somatic examination, basic biological tests, stool culture and parasitological stool examination were without abnormalities. Colonoscopy revealed a diffuse minimal inflammatory colitis interesting the entire colon, and the pathological examination of multiple biopsies led to the diagnosis of isolated LC. She was treated with 5-amino-salicylate with good evolution. Conclusion: Our observation is, to our knowledge the third reporting the association of LC with SLE; it is characterized in addition by the young age of occurrence. This association once again comforts the hypothesis of a common pathophysiology to these two diseases mainly involving genetic predisposition, HLA susceptibility, immune disorders, and environmental factors.
\end{abstract}

Keywords: lymphocytic colitis, systemic lupus erythematosus, microscopic colitis, lupus enteritis

Cite This Article: Salem Bouomrani, Souad Yahyaoui, Mouna Guermazi, and Nesrine Regaïeg, "Lymphocytic Colitis Associated to Systemic Lupus Erythematosus in a Young Woman." American Journal of Medical Case Reports, vol. 7, no. 11 (2019): 274-276. doi: 10.12691/ajmcr-7-11-3.

\section{Introduction}

Gastrointestinal symptoms are common in systemic lupus erythematosus (SLE) [1] and are often difficult to interpret: specific lesion of lupus? Infectious complication? Adverse effect of treatment? Or association with other dysimmune diseases? [1,2].

Gastrointestinal manifestations can be objectified in up to $50 \%$ of Lupus patients [3]; Specific lupus involvement (lupus enteritis, described for the first time in 1980 by Hoffman BI and Katz WA [4]), however, remains exceptional and unusual [5]. It typically affects the jejunum and ileum, whereas colonic and rectal involvement remains rare [5,6]. Indeed, only 11 among 288 patients with LES in the Iwamuro $\mathrm{M}$ et al series, had colic involvement (3.81\%) [6].

The mechanism involved is usually gastrointestinal vasculitis and/or gastrointestinal microthromboses, particularly if associated antiphospholipid antibody syndrome $[7,8]$. The most serious complication of this lupus colitis is colonic perforation, which is often fatal $[7,8]$.

More recently, other types of colitis have been reported in association with SLE suggesting other pathophysiological mechanisms [9]; the association SLE and lymphocytic colitis (LC) remains exceptional and unusual $[10,11]$.

We report an original observation of LC occurring in a 35-year-old lupus patient.

\section{Case Report}

35-year-old woman, having in her medical history a Hashimoto thyroiditis diagnosed for four years and a SLE diagnosed for three years.

The diagnosis of SLE with secondary antiphospholipid syndrome was retained based on the following data: distal polyarthritis without joint deformities or destruction, photosensitivity, malar erythema (vespertilio), right femoral vein thrombosis, leucopenia at $3100 / \mathrm{mm}^{3}$, positive anti-nuclear autoantibodies at $1 / 640$, positive native anti-DNA autoantibodies, and positive antiphospholipid autoantibodies (anti cardiolipin and anti- $\beta 2$ glucoprotein 1 ). She was treated with systemic corticosteroids, anticoagulant, and hydroxychloroquine with a favorable course. His Hashimoto's thyroiditis was stabilized with $125 \mu \mathrm{g} /$ day of thyroxine.

She consulted for fluid diarrhea that had been evolving for two months, not improved by symptomatic treatment, and associated for two weeks with rectorrhagia. 


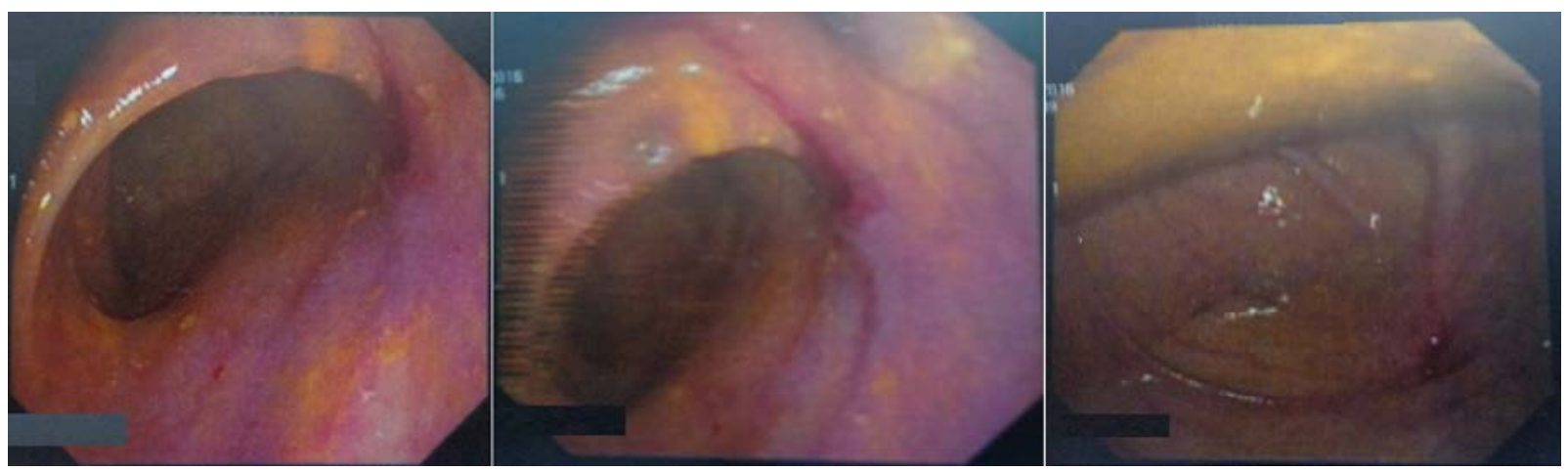

Figure 1. Colonoscopy: moderate inflammation of the colonic mucosa at different parts of the colon (transverse, right, and left colon)

The somatic examination was without abnormalities, the patient was particularly apyretic and without skin or joint active lesions of lupus disease. The abdomen was supple, depressible, painless on palpation, and without lymphadenopathy or visceromegaly.

The biological tests were within normal limits: erythrocyte sedimentation rate, C-reactive protein, serum protein electrophoresis, hemoglobin, leukocytes, platelets, calcemia, creatinine, ionogram, total cholesterol, triglycerides, liver enzymes, thyroid stimulating hormone, and glucose. Stool culture and parasitological stool examination were negative.

Colonoscopy revealed a colonic mucosa that was discreetly congestive and inflammatory, without ulceration, erosion, tumor lesions, diverticula or varicose veins. The appearance was that of a diffuse minimal inflammatory colitis interesting the entire colon (Figure 1).

Pathological examination of multiple biopsies led to the diagnosis of isolated LC. There were no micro-thromboses, granulomas or associated colonic vasculitis. She was treated with 5-amino-salicylate with good evolution.

\section{Discussion}

LC is a relatively new, rare, clinico-pathological entity that is still little known and often under-diagnosed in current medical practice. Its prevalence varies from 5.7/100,000 to $12.9 / 100,000$ depending on the series and countries [12,13]. It integrates with collagenous colitis in the nosological framework of microscopic colitis [12,13,14].

The etiology of these colitis is not yet known. It appears to be multifactorial involving genetic predisposition, immune dysfunction, drug intake, infections, and environmental factors $[12,13,14]$.

These colitis typically occur in the elderly, with an average age at diagnosis between 50 and 70 years [12,14] and subjects over 65 years old appear to be 5.6 times more exposed to these colitis compared with those under 45 years of age [15].

The main associations of LC are with thyroid disorders, celiac disease, and diabetes mellitus [12,13,14]. These associations suggest common genetic and immune factors $[12,13]$.

The association of microscopic colitis with SLE was reported for the first time by Desmoulins F et al in 1991 [9]; since only a few sporadic cases have been reported, mainly with collagenous colitis $[16,17,18]$. The hypothesis of a non-hazardous association is comforted by the observation of Al-kandari A et al, reporting SLE associated with collagen gastritis in a 47-year-old woman [19].

The association of CL and SLE remains exceptional and unusual; only two observations have been reported: a patient in the series of 104 microscopic colitis of Chande $\mathrm{N}$ et al, 30 of whom already had autoimmune associations $(0.96 \%)$ [10], and a second sporadic case reported by Hegazi MO and al [11].

The LC could thus be considered, for some authors, as a particular clinical presentation of lupus disease [11].

In addition, the presence in our observation of Hashimoto's thyroiditis is a further argument in favor of the non-hazardous association of these three diseases which shares a common dysimmunitary signature.

\section{Conclusion}

Our observation is, to our knowledge the third reporting the association of LC with SLE; it is characterized in addition by the young age of occurrence.

This association once again comforts the hypothesis of a common pathophysiology to these two diseases mainly involving genetic predisposition, HLA susceptibility, immune disorders, and environmental factors.

This association deserves to be well known by hospital practitioners because of its particular therapeutic and prognostic implications.

\section{Conflicts of Interest}

None.

\section{References}

[1] Luman W, Chua KB, Cheong WK, Ng HS. Gastrointestinal manifestations of systemic lupus erythematosus. Singapore Med J. 2001; 42(8): 380-4.

[2] Witt M, Zecher D, Anders HJ. Gastrointestinal manifestations associated with systemic lupus erythematosus. Eur J Med Res. 2006; 11(6): 253-60.

[3] Jaspersen D. Gastrointestinal manifestations of systemic lupus erythematosus: symptoms, diagnosis and differential diagnosis. Fortschr Med. 1992; 110(10): 167-9.

[4] Hoffman BI, Katz WA. The gastrointestinal manifestations of systemic lupus erythematosus: a review of the literature. Semin Arthritis Rheum. 1980; 9: 237-247. 
[5] Smith LW, Petri M. Lupus enteritis: an uncommon manifestation of systemic lupus erythematosus. J Clin Rheumatol. 2013; 19(2): 84-6.

[6] Iwamuro M, Okada H, Kato J, Tanaka T, Sada KE, Makino H, et al. Indications for colonoscopy in patients with systemic lupus erythematosus. Hepatogastroenterology. 2013; 60(128): 1940-4.

[7] Zizic TM, Shulman LE, Stevens MB. Colonic perforations in systemic lupus erythematosus. Medicine (Baltimore). 1975; 54(5): 411-26.

[8] Ho MS, Teh LB, Goh HS. Ischaemic colitis in systemic lupus erythematosus--report of a case and review of the literature. Ann Acad Med Singapore. 1987; 16(3): 501-3.

[9] Desmoulins F, Lévy P, Boccaccio F, Thulliez M, Christoforov B. Collagenous colitis associated with systemic lupus erythematosus: first case. Gastroenterol Clin Biol. 1991; 15(4): 368.

[10] Chande N, Driman DK, Reynolds RP. Collagenous colitis and lymphocytic colitis: patient characteristics and clinical presentation. Scand J Gastroenterol. 2005; 40(3): 343-7.

[11] Hegazi MO, Owayed SF, Mourou M, Joneja M, Mashankar A. Lymphocytic enterocolitis in systemic lupus erythematosus. Saudi J Gastroenterol. 2009; 15(4): 274-6.

[12] Pardi DS. Diagnosis and Management of Microscopic Colitis. Am J Gastroenterol. 2017; 112(1): 78-85.
[13] Chetty R, Govender D. Lymphocytic and collagenous colitis: an overview of so-called microscopic colitis. Nat Rev Gastroenterol Hepatol. 2012; 9(4): 209-18.

[14] Park T, Cave D, Marshall C. Microscopic colitis: A review of etiology, treatment and refractory disease. World J Gastroenterol. 2015; 21(29): 8804-10.

[15] Williams JJ, Kaplan GG, Makhija S, Urbanski SJ, Dupre M, Panaccione R, et al. Microscopic colitis-defining incidence rates and risk factors: a population-based study. Clin Gastroenterol Hepatol. 2008; 6(1): 35-40.

[16] Heckerling P, Urtubey A, Te J. Collagenous colitis and systemic lupus erythematosus. Ann Intern Med. 1995; 122(1): 71-2.

[17] Bachevalier F, Lederlin P, Laugros A, Durr JF, Le Quang D. Collagenous colitis associated with systemic lupus erythematosus and circulating anticoagulant syndrome. Rev Med Interne. 1997; 18(11): 908-9.

[18] Alikhan M, Cummings OW, Rex D. Subtotal colectomy in a patient with collagenous colitis associated with colonic carcinoma and systemic lupus erythematosus. Am J Gastroenterol. 1997; 92(7): 1213-5.

[19] Al-Kandari A, Al-Alardati H, Sayadi H, Al-Judaibi B, Mawardi M. An unusual case of collagenous gastritis in a middle- aged woman with systemic lupus erythromatosis: a case report. J Med Case Rep. 2014; 8: 278.

(C) The Author(s) 2019. This article is an open access article distributed under the terms and conditions of the Creative Commons Attribution (CC BY) license (http://creativecommons.org/licenses/by/4.0/). 